\title{
PERFIL DO ESTILO DE VIDA DE CONTROLADORES DE TRÁFEGO AÉREO: UM ESTUDO DE CASO
}

\section{Lifestyle profile of controllers of air traffic: a case study}

\author{
Thiago Ferreira de Sousa'; Sueyla Ferreira da Silva dos Santos ${ }^{2}$
}
${ }^{1}$ Programa de Pós-Graduação da Universidade Federal de Santa Catarina (UFSC), Grupo de Pesquisa em Atividade Física e Saúde (GPAF), Núcleo de Pesquisa em Atividade Física e Saúde (NuPAF), Centro de Desportos, Florianópolis, Brasil
${ }^{2}$ Programa de Pós-Graduação em Saúde Coletiva pela Universidade Estadual do Sudoeste da Bahia (UESB), Grupo de
Pesquisa em Atividade Física e Saúde, Salvador, Bahia

\begin{abstract}
Resumo: O estilo de vida está diretamente relacionado com a autopercepção de bem-estar geral, além de ser um importante componente da qualidade de vida. Sendo assim, hábitos de vida considerados saudáveis podem estar relacionados a menores chances de doenças cardiovasculares ou metabólicas, especialmente em trabalhadores. Considerando tais aspectos, o objetivo do presente estudo foi descrever as características do estilo de vida de um grupo de controladores do tráfego aéreo de uma cidade litorânea do sul da Bahia, Brasil. Para tanto, foi realizado um estudo de caso de delineamento transversal em um grupo de militares responsável pelo controle do tráfego aéreo, nas posições de torre de controle e controle de aproximação. Como instrumento de pesquisa foi utilizado o questionário Perfil do Estilo de Vida Individual, que contempla as seguintes características do estilo de vida: prática de atividade física, nutrição, relacionamentos, comportamento preventivo e controle do stress. O instrumento avalia cada item dos componentes do estilo de vida por meio de uma escala likert de quatro opções de resposta. Considerou-se como perfil negativo os escores 0 e 1 e como perfil positivo os escores 2 e 3 . Para a apresentação dos dados foram utilizados procedimentos de estatística descritiva. Observou-se perfil negativo para todos os itens do componente nutrição e para um componente da atividade física, sendo que para o componente relacionamentos foi identificado um item com escore máximo (perfil positivo). A adoção de hábitos saudáveis é um importante elemento para a prevenção de possíveis doenças crônicas não-transmissíveis, assim, sugere-se a implementação de programas de promoção de saúde para trabalhadores.
\end{abstract}

Palavras-chave: Estilo de vida, Conduta de saúde, Trabalhadores.

Abstract: The lifestyle is directly related the perception of wellness, as an important component of quality of life. Thus, life habits healthy may be related to lower risk of cardiovascular disease or metabolic disorders, especially among workers. The purpose of this study was to describe the characteristics of the lifestyle of a group of air traffic controllers in a town in southern of Bahia, Brazil. The was carried out a study (cross-sectional) with a group of soldiers responsible for air traffic control, in positions of control tower and approach control. Was used the instrument Individual Lifestyle Profile with purpose of identified the characteristics of lifestyle (physical activity, nutrition, relationships, preventive behavior and control of stress). The instrument evaluates each item of the components of the lifestyle through a likert scale of four options for response. Was considered as negative profile scores 0 and 1 and positive profile as scores 2 and 3 . The data are presented as means and frequency of the group. Identified negative profile for all items of nutrition component and a component of physical activity, for a component of relationships was identified an item as maximum score (positive profile). The adoption of healthy habits is an important element for the prevention of possible non-transmissible chronic diseases, so it is suggested the implementation of programs of health promotion for employees.

Keywords: Lifestyle, Behavior, Workers.

Aceito em 22/09/2009 - Rev. Educ. Fís. 2009 - 19-25. Rio de Janeiro - RJ - Brasil

\section{INTRODUÇÃO}

O estilo de vida pode ser entendido como o conjunto de ações habituais que refletem as atitudes, valores e oportunidades das pessoas $^{(1)}$, composto por cinco componentes: atividade física, nutrição, relacionamentos, comportamento preventivo e controle do stress $^{(2)}$. Destaca-se que a adoção de hábitos negativos de vida demonstra relação com o surgimento de doenças crônicas nãotransmissíveis, em especial as doenças cardiovasculares $^{(3)}$, sendo esta uma preocupação para a saúde pública.

Os estudos de base populacional(4), assim como os estudos com trabalhadores ${ }^{(5,6)}$, que 
apresentam como foco o monitoramento de hábitos negativos demonstraram que apesar das constantes informações sobre os benefícios derivados de um estilo de vida saudável, uma parcela das pessoas não adere a uma proposta de vida saudável.

Sendo assim, a análise do perfil do estilo de vida em trabalhadores representa um importante foco em pesquisas, principalmente para o diagnóstico de informações que possam contribuir com iniciativas relacionadas à saúde do trabalhador. Em subgrupos específicos, como militares, o conhecimento acerca dos componentes dos hábitos de vida é essencial ${ }^{(7)}$, haja vista uma ênfase em estudos relacionados à descrição de apenas a aptidão física destes profissionais ${ }^{(8,9)}$.

Entre os controladores de tráfego aéreo, grupo que atua em situação de vulnerabilidade ao estresse e condições de extrema atenção, destaca-se a importância de oferta de estratégias de apoio e motivação para a adoção e manutenção do estilo de vida. Aspectos como aptidão muscular e resistência em níveis não adequados, bem como excesso de peso corporal, são fatores de risco prevalentes e apresentam relação com a produtividade e conseqüente qualidade de vida desses profissionais ${ }^{(10)}$.

O objetivo deste estudo foi descrever as características do estilo de vida dos militares controladores do tráfego aéreo, de uma cidade litorânea do interior da Bahia.

\section{METODOLOGIA}

O presente estudo caracteriza-se como descritivo de delineamento transversal, do tipo estudo de caso. Foram obtidas as informações dos 10 controladores de vôo que atuam em um município da região do sul do Estado da Bahia (Brasil). Todos os sujeitos são do sexo masculino e militares que exercem a função de controladores de tráfego aéreo nas posições referente à torre de controle e controle de aproximação.

A coleta de dados foi realizada em janeiro de 2006 no local de trabalho desses funcionários, com horário previamente agendado. Para a coleta de dados foi utilizado o instrumento Perfil do Estilo de Vida Individual - $P E V^{(2)}$ e um instrumento sobre aspectos demográficos, ambos aplicados de maneira auto-reportada na forma livre. O $P E V I$ foi recentemente avaliado quanto às suas capacidades psicométricas, e constatouse que o instrumento apresenta medidas confiáveis ${ }^{(11)}$.

O PEVI apresenta cinco componentes: nutrição, atividade física, comportamento preventivo, relacionamentos e controle do stress e cada componente é composto por três itens (perguntas). O instrumento PEVI é apresentado no QUADRO 1.

\section{QUADRO 1 \\ COMPONENTES E ITENS DO INSTRUMENTO PEV|(2)}

Nutrição:

a) Sua alimentação diária inclui pelo menos 5 porções de frutas e hortaliças.

b) Você evita ingerir alimentos gordurosos (carnes gordas, frituras) e doces.

c) Você faz 4 a 5 refeições variadas ao dia, incluindo café da manhã completo.

Atividade física:

d) Você realiza ao menos 30 minutos de atividades físicas moderadas/intensas, de forma contínua ou acumulada, 5 ou mais dias na semana.

e) Ao menos duas vezes por semana você realiza exercícios que envolvam força e alongamento muscular.

f) No seu dia-a-dia, você caminha ou pedala como meio de transporte e, preferencialmente, usa as escadas ao invés do elevador.

Comportamento preventivo:

g) Você conhece sua pressão arterial, seus níveis de colesterol e procura controlá-los.

h) Você não fuma e não ingere álcool (ou ingere com moderação).

i) Você respeita as normas de trânsito (como pedestre, ciclista ou motorista); se dirige, usa sempre o cinto de segurança e nunca ingere álcool.

Relacionamentos:

j) Você procura cultivar amigos e está satisfeito com seus relacionamentos.

k) Seu lazer inclui encontros com amigos, atividades esportivas em grupo, participação em associações ou entidades sociais.

I) Você procura ser ativo em sua comunidade, sentido-se útil no seu ambiente social.

Controle do stress:

m) Você reserva tempo (ao menos 5 minutos) todos os dias para relaxar.

n) Você mantém uma discussão sem alterar-se, mesmo quando contrariado.

o) Você equilibra o tempo dedicado ao trabalho com o tempo dedicado ao lazer. 
As respostas de cada item variam em uma escala likert de quatro opções e para cada opção é atribuída uma pontuação com variação de zero a três pontos. As respostas e seus respectivos pontos são: absolutamente não faz parte do seu estilo de vida = 0 ; às vezes corresponde ao seu comportamento $=1$; quase sempre verdadeiro no seu comportamento $=2$; a afirmação é sempre verdadeira no seu dia-a-dia; faz parte do seu estilo de vida $=3$.

Para análise do PEVI utiliza-se a média aritmética dos valores de cada item dos componentes para análise do perfil do estilo de vida do grupo, sendo considerado como Perfil Negativo os escores zero e um (01) e Perfil Positivo os escores dois e três ${ }^{(2)}$. O autor $^{(2)}$ do instrumento propõe a representação visual das características do estilo de vida em uma estrela de cinco pontos, que formam o pentáculo do bem-estar (FIGURA 1), seguindo as seguintes orientações: deixar em branco se marcou zero para o item; preencher do centro até o primeiro círculo se marcou 1; preencher do centro até o segundo círculo se marcou 2; preencher do centro até a extremidade final se marcou 3.

FIGURA 1

REPRESENTAÇÃO VISUAL PROPOSTA PARA APRESENTAÇÃO DO PERFIL DO ESTILO DE VIDA

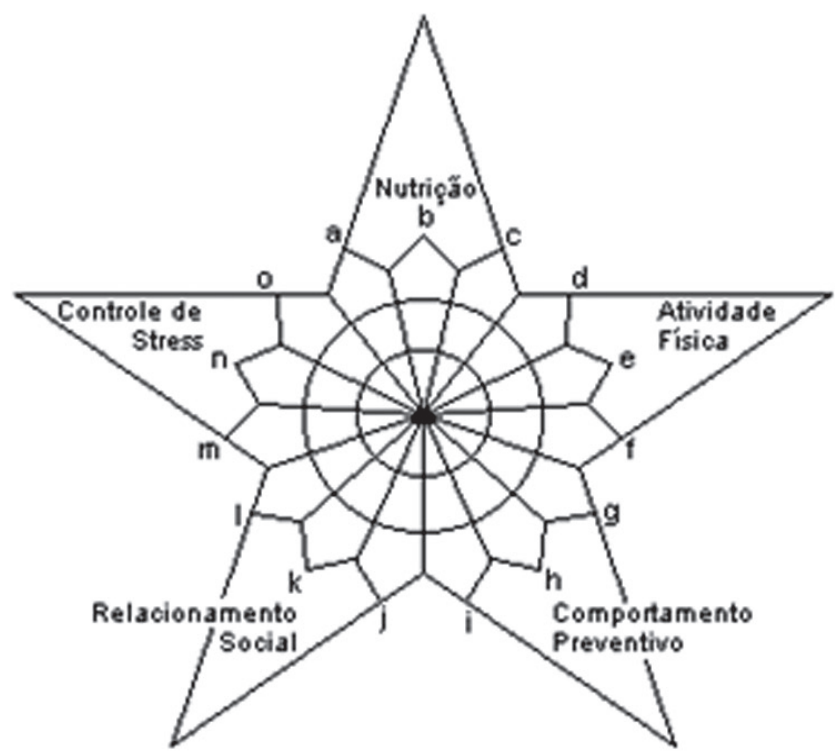

Em relação ao instrumento sobre as informações demográficas utilizou-se um questionário com perguntas de opções de respostas abertas e fechadas. Os indicadores e opções de resposta foram: idade (pergunta aberta do tipo quantitativa discreta); estado civil (solteiro e casado ou vivendo com parceiro); número de filhos (nenhum filho, um filho e dois ou mais filhos), escolaridade (ensino médio completo, ensino superior incompleto e ensino superior completo) e tempo de serviço (um a cinco anos, seis a dez anos, 11 a 19 anos e 20 anos ou mais).

Foram empregados os procedimentos de estatística descritiva (freqüência absoluta, média com aproximação decimal para os valores iguais ou superiores a cinco, mínimo e máximo) para a apresentação das informações. Os tratamentos estatísticos foram realizados no software estatístico SPSS versão 16.0. Todos os sujeitos envolvidos foram informados sobre os objetivos do estudo e o sigilo do tratamento dos dados, essas informações foram disponibilizadas aos controladores de vôo de forma semelhante. A coleta de dados somente iniciou após a assinatura do Termo de Consentimento Livre e Esclarecido (TCLE) que consta em anexo, sendo esse um dos procedimentos constantes na Resolução 196/96, acerca da realização de pesquisas com seres humanos.

\section{RESULTADOS}

A idade média dos controladores de vôo foi de 32,9 anos, com variação de 21 a 41 anos. As informações relativas às características demográficas são apresentadas na TABELA 1.

TABELA 1

CARACTERÍSTICAS DEMOGRÁFICAS DOS CONTROLADORES DE VÔO, BAHIA. 2006.

\section{Variáveis}

Freqüência (n)

Estado Civil

Solteiro 3

Casado 7

Número de Filhos

Não tem filhos 3

Um filho 4

Dois ou mais filhos 3

\section{Escolaridade}

Ensino médio completo 4

Ensino superior incompleto 4

Ensino superior completo 2

Tempo de serviço

1 a 5 anos 2

6 a 10 anos 3

11 a 19 anos 4

20 anos ou mais $\quad 1$ 
Os escores médios das características do estilo de vida dos controladores de vôo estão apresentados na TABELA 2, além disso, a representação visual dessesescoreséapresentada por meio do pentáculo do bem-estar na FIGURA 1. Observou-se em relação ao componente nutrição uma maior predominância do escore 1 , sendo este o componente que demonstrou um perfil negativo superior quando comparado aos demais componentes. Entretanto, os seguintes componentes do estilo de vida dos controladores de vôo apresentaram perfil positivo (escore $=2$ ou 3): comportamento preventivo, relacionamentos, controle do stress e atividade física.

Destaca-se que no componente atividade física, o grupo apresentou escore 1 para o item "d", referente à prática de atividade física de forma moderada / intensa por um mínimo de 30 minutos em cinco ou mais dias na semana. Além disso, o maior escore identificado foi para o item "j" do componente relacionamento, que se refere à satisfação com os relacionamentos e o cultivo das amizades.

TABELA 1

CARACTERÍSTICAS DEMOGRÁFICAS DOS CONTROLADORES DE VÔO, BAHIA. 2006.

Variáveis

Média

Nutrição
a)
b)
1
c)
1

Atividade física

d)

1

e)

2

f)

2

Comportamento preventivo

g)

2

h)

2

i)

2

Relacionamentos

j)

k)

2

l)

Controle do stress

$\mathrm{m})$

n)

o)
FIGURA 2

REPRESENTAÇÃO VISUAL DO PERFIL DO ESTILO DE VIDA DOS CONTROLADORES DE VÔO.

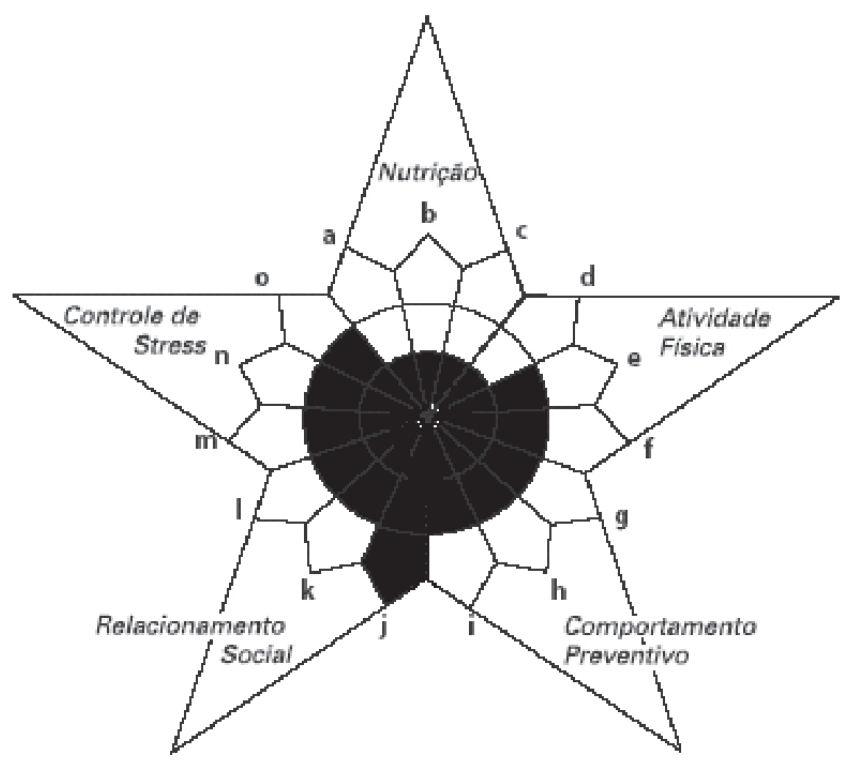

DISCUSSÃO

Este estudo de caso apresenta como limitações a utilização de um questionário para obtenção das informações sobre o estilo de vida, contudo, o instrumento aplicado apresenta capacidades psicométricas para utilização com este fim. O ponto forte deste estudo está na população de sujeitos participantes, os controladores de tráfego aéreo de uma cidade litorânea do sul da Bahia, e, principalmente, pela região que fazem parte.

Tendo em vista a relevância dos aspectos do estilo de vida para a qualidade de vida, destaca-se que, de uma maneira geral, os sujeitos investigados demonstraram um perfil de estilo de vida positivo. Essa informação é importante, haja vista a relação direta de um estilo de vida negativo com doenças e mortalidade ${ }^{(3)}$. Entretanto, as informações identificadas no presente estudo quando comparadas a outros estudos devem ser cautelosas, principalmente pelo número pequeno de sujeitos envolvidos.

Dos cinco componentes do estilo de vida propostos pelo instrumento, destaca-se que o componente relativo aos hábitos alimentares (nutrição) apresentou escores negativos em todos os itens. Observa-se que a adoção 
de uma alimentação saudável, por meio do consumo de frutas e verduras não tem sido prevalente na população brasileira ${ }^{(12)}$. O sistema de vigilância epidemiológica nacional $^{(4)}$ recentemente observou que a prevalência de consumo regular de frutas e verduras, em cinco ou mais dias durante a semana nas capitais brasileiras variou entre $19,5 \%$ em Belém a 41,6\% em Florianópolis, em relação à capital do estado da Bahia, Salvador, a prevalência foi de $27,7 \%$. Essas observações enfatizam, assim como no presente estudo, que a população brasileira apresenta hábitos considerados não adequados quanto ao consumo de frutas e verduras.

Em relação a trabalhadores, outros estudos demonstraram prevalências superiores para o consumo de frutas e verduras em níveis reduzidos ${ }^{(5,13)}$. Contudo, trabalhadores da indústria alemã apresentaram consumo de alimentos relacionados a uma alimentação saudável em níveis adequados(14). Esta maior diferença pode ocorrer em função ao estímulo a adoção de hábitos saudáveis por meio de programas relacionados à alimentação saudável ofertados para estes trabalhadores.

No entanto, estudos demonstraram que programas destinados à melhoria dos hábitos alimentares, por meio da oferta de alimentação ao trabalhador podem não estar sendo eficientes, contribuindo assim com um impacto negativo na saúde do trabalhador, além do aumento dos casos de obesidade nessa população(15,16).

Pois, um dos fatores que contribui para o excesso de peso corporal são os alimentos hipercalóricos, ricos em gorduras saturadas, que estão relacionados com o surgimento das hiperlipidemias, e possíveis doenças cardiovasculares $^{(3)}$. O excesso de peso corporal representa um importante problema de saúde pública em diferentes nações, e que também tem sido diagnosticado em subgrupos como em militares ${ }^{(17)}$.

Quanto ao componente atividade física, os controladores de vôo apresentaram perfil negativo para a prática de atividade física aeróbia em nível moderado à intensa, de forma contínua ou acumulada, em pelo menos cinco dias na semana. Vale salientar que outro estudo realizado com trabalhadores demonstrou o não alcance das recomendações mínimas de prática de atividade física aeróbia(18), porém não corrobora em estudo realizado com militares que apresentaram a regularidade da prática de exercícios físicos ${ }^{(7)}$.

Para a população da cidade de Salvador (Bahia), a prevalência de inatividade física em relação aos quatro domínios (lazer, atividades domésticas, laborais e deslocamento ativo) foi de $24,6 \%{ }^{(4)}$. Além disso, recentes informações sobre a inatividade física no lazer em trabalhadores da indústria demonstraram prevalência de $48,3 \%$ para os trabalhadores da região nordeste, sendo esta a região que apresentou prevalência superior de inatividade física quando comparado as demais regiões do Brasil(19). Embora a relevância de um estilo de vida saudável para a saúde, King et al. ${ }^{(20)}$ demonstraram que a aderência a estilos de vida saudáveis declinaram nos últimos 18 anos, entre 1988 a 2006, sendo tais informações provenientes de estudos realizados com a população dos Estados Unidos.

\section{CONCLUSÃO}

Conclui-se que os hábitos alimentares e um dos itens da prática de atividade física são os principais componentes que apresentaram perfil negativo do estilo de vida dos controladores de tráfego aéreo, e os relacionamentos os que apresentaram perfil positivo. Nota-se a necessidade de se equilibrar o tempo dedicado ao lazer e ao trabalho, assim como as condições de trabalho diante a influência que esses fatores exercem sobre a qualidade de vida destes profissionais. Além disso, sugerese a realização de programas de incentivo à prática de atividade física e hábitos alimentares saudáveis em militares, assim como para os trabalhadores da região nordeste do Brasil.

Além disso, faz-se necessário a condução de outros estudos com delineamento diferenciado desse e que possam englobar 
um número maior destes profissionais, assim como de outros estados do país. Tendo em vista que a caracterização dos componentes do estilo de vida e a sua relação com outros fatores associados representam importantes informações para o desenvolvimento de medidas voltadas à saúde e à qualidade de vida desses profissionais.

\section{AGRADECIMENTOS}

Aos militares que participaram do presente estudo e ao professor Marcílio Moraes pelo auxílio na fase de coleta de dados.

\section{REFERÊNCIAS BIBLIOGRÁFICAS}

1. Nahas MV, Barros MVG, Francalacci V. O pentáculo do bem-estar - base conceitual para avaliação do estilo de vida de indivíduos ou grupos. Revista Brasileira de Atividade Física \& Saúde 2000; 5: 48-59.

2. Nahas MV. Atividade Física, Saúde e Qualidade de Vida: conceitos sugestões para um estilo de vida ativo. 4 ed. Londrina: Midiograf, 2006.

3. World Health Organization. The World Health Report 2002. Reducing risks, promoting healthy life. Geneva: World Health Organization, 2002.

4. Brasil. Ministério da Saúde. Vigilância de Fatores de Risco e Proteção para Doenças Crônicas por Inquérito Telefônico: estimativas sobre freqüência e distribuição sócio-demográfica de fatores de risco e proteção para doenças crônicas nas capitais dos 26 Estados brasileiros e no Distrito Federal em 2006. Brasília, Ministério da Saúde, 2009. 114 p.

5. Barros MVG, Nahas MV. Comportamento de risco, auto-avaliação do nível de saúde e percepção de estresse entre trabalhadores da indústria. Rev Saúde Pública 2001; 35: 554-63.

6. Fonseca, S. A. Inatividade física no lazer e outros fatores de risco à saúde em industriários catarinenses, 1999-2004. 2005. 100 f. Dissertação (Mestrado em Educação Física) - Centro de Desportos. Universidade Federal de Santa Catarina, Florianópolis, 2005.
7. Pizzi C, Evans SA, Stavola BL, Evans A, Clemens F, Santos Silva I. Lifestyle of UK commercial aircrews relative to air traffic controllers and the general population. Aviat Space Environ Med 2008; 79: 964-74.

8. Pereira EF, Teixeira CS. Propostas de valores normativos para avaliação da aptidão física em militares da Aeronáutica. Rev Bras Educ Fis Esp 2006; 20: 249-56.

9. Oliveira EAM, Anjos LA. Medidas antropométricas segundo aptidão cardiorrespiratória em militares da ativa, Brasil. Rev Saúde Pública 2008; 42: 217-23.

10. Kyröläinen $H$, Häkkinen $K$, Kautiainen $H$, Santtila M, Pihlainen K, Häkkinen A. Physical fitness, BMI and sickness absence in male military personnel. Occup Med 2008; 58: 251-56.

11. Both J, Borgatto AF, Nascimento JV, Sonoo CN, Lemos CAF, Nahas MV. Validação da escala "Perfil do Estilo de Vida Individual". Revista Brasileira de Atividade Física \& Saúde 2008; 13: 5-14.

12. Jaime PC, Monteiro CA. Fruit and vegetable intake by Brazilian adults, 2003. Cad Saúde Pública 2005; 21: S19-S24.

13. Oliveira ESA. Atividade física habitual e outros comportamentos relacionados à saúde dos servidores da Universidade Federal de Santa Catarina: tendência secular 1994-2004. 2005. 94 f. Dissertação (Mestrado em Educação Física) Centro de Desportos. Universidade Federal de Santa Catarina, Florianópolis, 2005.

14. Reime B, Novak P, Born J, Hagel E, Wanek V. Eating habits, health status, and concern about health: a study among 1641 employees in the German Metal Industry. Prev Med 2000; 30: 295-301.

15. Veloso IS, Santana VS. Impacto nutricional do programa de alimentação do trabalhador no Brasil. Rev Panam Salud Pública 2002; 11: 24-3.

16. Veloso IS, Santana VS, Oliveira NF. Programas de alimentação para o trabalhador e seu impacto sobre ganho de peso e sobrepeso. Rev Saúde Pública 2007; 41: 769-76. 
17. Neves EB. Prevalência de sobrepeso e obesidade em militares do exercito brasileiro: associação com a hipertensão arterial. Cien Saúde Colet 2008; 13: 1661-68.

18. Yancey AK, Mccarthy WJ, Taylor WC, Merlo A, Gewa C, Weber MD et al. The Los Angeles Lift Off: a sociocultural environmental change intervention to integrate physical activity into the workplace. Prev Med 2004; 38: 848-56.

19. Nahas MV. Estilo de Vida e Hábitos de Lazer dos Trabalhadores das Indústrias Brasileiras. Relatório Geral. SESI, 2009.
20. King DE, Mainous AG, Carnemolla M, Everett CJ. Adherence to Healthy Lifestyle Habits in US Adults, 1988-2006. Am J Med 2009; 122: 528-34.

Endereço para correspondência:

Thiago Ferreira de Sousa

Campus Universitário, Coordenadoria de PósGraduação em Educação Física.

CEP: 88040-900

Bairro Trindade, Florianópolis, Santa Catarina.

Telefone: +55 (48) 3721.8519

Email: tfsousa_thiago@yahoo.com.br 